\title{
FTIR spectroscopy combined with chemometric: a versatile tool for quality evaluation of fried vermicelli
}

\author{
Taimoor Hassan Shaikh, Sarfaraz Ahmed Mahesar, \\ Syed Nasrullah Shah, Abdul Hameed Kori, \\ Syed Tufail Hussain Sherazi, Saeed Ahmed Lakho
}

\author{
National Centre of Excellence in Analytical Chemistry, \\ University of Sindh, Pakistan
}

Keywords:

Fried vermicelli

Soxhlet extraction

Fatty acids

GC-MS

FTIR

Chemometrics

Article history:

Received 16.01.2017

Received in revised

form 19.03.2017

Accepted 30.03.2017

\section{Corresponding}

author:

Sarfaraz Ahmed

Mahesar

E-mail:

sarfaraz.mahesar@

usindh.edu.pk

DOI: $10.24263 / 2304-$

974X-2017-6-1-8

\section{Abstract}

Introduction. Snacks are common food throughout the world including subcontinent. Most of the snack foods are prepared in oil and fat. In current study, fried vermicelli (common snack food) was selected to evaluate the quality as it is largely consumed in many countries.

Materials and methods. Fried vermicelli was subjected for the extraction of total oil by applying Soxhlet extraction method using hexane as a solvent. Fatty acid composition of extracted fried vermicelli oil was checked on GC-MS. Similarly, FTIR spectroscopy was also used to record the spectra of same oil for the development of simple methodology to quantify fatty acid groups and ratios.

Results and discussion. High percentage of oil content was observed in all fried vermicelli samples (19.77-32.99\%). Fatty acid composition exposed that palmitic (34.6-47.5\%), stearic (4.76 to $10.6 \%$ ), oleic $(27.2-37.0 \%)$ and elaidic acid (12.0$24.3 \%$ ) were predominant fatty acids among saturated and monounsaturated fatty acids. While polyunsatured fatty acids were observed comparatively in less quantity in fried vermicelli (0.66 to $5.99 \%)$. The presence of higher trans fatty acids indicated that fried vermicelli was prepared in hydrogenated oil. Fatty acid ratios of some important groups were observed in the range of $0.72-1.92$ SFA/UFA, $0.013-0.130$ cis PUFA/SFA, 0.1 to 1.81 trans FA/cis FA, 0.01 to 0.097 cis PUFA/SFA + TFA, 27.7-37.05 cis MUFA + cis PUFA/SFA + TFA. The obtained results of groups and ratios by GC-MS were used to develop different calibration models for the quantification of fatty acid groups and fatty acid ratios using PLS chemometric approach. The developed PLS models using selected wavelength regions showed higher correlations $(>0.99)$ with GC-MS results.

Conclusions. It can be concluded that quality of fried vermicelli marketed locally is worrisome matter for the consumers and quality control authorities. FTIR provided complete profiling of groups and ratios in fried vermicelli very accurately with negligible difference in the results. The proposed methodology serves as a rapid and simple quantitative tool for quality evaluation of major groups and ratios. 


\section{Introduction}

Nutritionists are interested in the fatty acid intake, as the oil and fats play an essential role in human nutrition as energy source and metabolic activities since it is a major research focus in nutritional sciences. They regulate the physiological process in human body structure and produce energy [1].

Currently, nutritionists recommend intake of saturated fatty acids (SFA), monounsaturated fatty acids (MUFA) and polyunsaturated fatty acids (PUFA) in a balanced amount for a nutritious diet because higher intake of SFA has been associated with cardiovascular diseases (CVD), whereas MUFA and PUFA consumption is related with beneficial health effects [2]. MUFA and PUFA substituted SFA [3] because MUFA lower the risk factors of CVD [4]. PUFA can be linked to negative or beneficial health effects [5]. The epidemiological research on clinical role of $n-6 / n-3$ ratio of PUFA was studied and found an increased risk of cardiovascular and coronary heart diseases [6]. Conversely, PUFA/SFA ratio is associated with CVD which can be lower by replacing SFA with PUFA in a balanced diet [7] as the increased intake of PUFA has beneficial effects [8]. However, some detrimental properties are also there, unsaturated FA can be reacted with oxygen especially in air which makes them rancid [9]. The process of partial hydrogenation is used to avoid the rancidity of unsaturated FA [10]. Before hydrogenation process, unsaturated FA present in cis configuration, whereas partial hydrogenation converts some of the cis double bonds into its trans configuration and relocate the position of double bonds [11]. WHO scientific review committee on trans fat recommend daily intake of partially hydrogenated oil should be less than $1 \%$ [12]. FDA proposed nutritional facts labeling rules to separate the trans fat from total fat content, which was effective from 1st January 2006 [11]. FDA remove partial hydrogenated oil which is the primary source of trans fat, from the list of GRAS (Generally recognized as safe) and allow food companies to eliminate the partially hydrogenated oils by reformulating their products by 2018 [13].

In Pakistan, no any study has been reported on the fatty acids as well as trans fatty acid contents of fried vermicelli, as it is a very popular snack food in sub-continent. GCMS is considered as an authentic technique for the quantification of fatty acid composition. The current study is conducted to investigate the main classes of fatty acid present in different fried vermicelli samples collected from different locations of Hyderabad, Sindh. The results of GC-MS correlated with the specific regions of FTIR spectra using PLS chemometric technique.

\section{Materials and methods}

\section{Chemicals and reagents}

Solvents and chemicals, such as hexane, methanol and potassium hydroxide (analytical grade) purchased from Merck (Darmstadt, Germany).

\section{Sample collection}

18 samples of fried vermicelli were collected from different local shops of Hyderabad, Pakistan. 


\section{Oil extraction}

Fried vermicelli samples were subjected for the extraction of total fat and oil by applying Soxhlet extraction method using hexane as a solvent [14]. Total oil content was calculated as percentage mass of the sample. Extracted fried vermicelli oil was transferred into vials and kept at $-4{ }^{\circ} \mathrm{C}$ until further analysis.

\section{Determination of fatty acid composition}

Fatty acid composition of fried vermicelli was analyzed by preparing Fatty acid methyl ester (FAME) using IUPAC standard method [15]. GC-MS chromatograms of fried vermicelli were recorded via Agilent GC-MS. Obtained data was analyzed using ChemStation software. For identification of fatty acids in fried vermicelli, NIST and Wiley libraries were used to compare the fatty acids by matching $>90 \%$ similarity.

\section{Gas chromatography - mass spectrometry conditions}

FAME were analyzed on gas chromatography (Agilent $6890 \mathrm{~N}$ ) coupled with a mass selective detector (Agilent MS-5975) and an auto sampler injector (Agilent 7683-B) (Little Fall, NY, USA). For the separation of FAME, 5\% phenyl methylsiloxane (HP-5MS) capillary column having $30 \mathrm{~m}$ length with internal diameter of $0.25 \mathrm{~mm}$ from Agilent Technologies (Palo Alto, CA, USA) was used. Initial oven temperature $150 \mathrm{oC}$ was set for $2 \mathrm{~min}$ and ramped at $4 \mathrm{C} / \mathrm{min}$ up to $230{ }^{\circ} \mathrm{C}$ and hold for $5 \mathrm{~min}$. Helium was used as a carrier gas with flow rate of $1 \mathrm{ml} / \mathrm{min}$ and a split ratio 1:50. Temperature of injector and detector was set at $240{ }^{\circ} \mathrm{C}$ and $260{ }^{\circ} \mathrm{C}$, respectively. Electron impact (EI) ionization source at $70 \mathrm{ev}$ in mass spectrometer was used with the scan range of $50-550 \mathrm{~m} / \mathrm{z}$ as reported in earlier study [16].

\section{Statistical analyses and calculations}

Peaks were identified by GC-MS libraries of NIST and Wiley and percentage of fatty acids were quantified by area percent method.

\section{Fourier Transform Infrared spectroscopy}

All the infrared spectra of Fried vermicelli samples were recorded using Nicolet 320 Fourier transform infrared (FTIR) spectrometer (Thermo Nicolet Analytical Instruments) Madison, WI, USA with ZnSe Single bounce ATR accessory (Spectra-Tech, Shelton, CT) with $\mathrm{KBr}$ beam splitter and deuterated triglycine sulphate (DTGS) detector. OMNIC software (version 7.3) was used for the instrumental control and acquisition of data. 24 accumulated scans with the resolution of $4 \mathrm{~cm}^{-1}$ in the range of $4000-650 \mathrm{~cm}^{-1}$ were set for recording spectra. Background spectrum was collected before acquiring each spectrum of sample. Soft tissue and hexane were used for the cleaning of ZnSe crystal after every sample.

\section{Results and discussion}

Snack food products are of wider interest for food researchers because of its high consumption. Fried vermicelli is one of the common snack food in sub-continent especially in Pakistan. It is a type of pasta, round in section and somewhat thinner than 
spaghetti. It is prepared using different recipes and looks like thin vermicelli usually roasted/fried in oil/fat until light brown color appears. Initially, the samples were subjected to oil extraction using Soxhlet extractor to know the exact oil content. High percentage of oil content was observed in all fried vermicelli samples (19.77 and 32.99\%). The highest amount of oil was extracted from fried vermicelli-14 and minimum quantity was found in fried vermicelli-18. Table 1 shows the fatty acids composition of fried vermicelli expressed in percentage. In analyzed samples, palmitic (C-16:0) and stearic acid (C-18:0) were present in higher amounts, whereas dodecanoic (C-12:0), myristic (C14:0) and arachidic acid (C-20:0) found in relatively low quantities. Among SFA, palmitic acid in fried vermicelli was present in the range of $34.6-47.5 \%$. The stearic acid in fried vermicelli was present in the range of 4.76-10.6\%. Oleic acid was observed as main contributor among MUFA in fried vermicelli samples. The percentage of oleic acid was found between $27.2-37.0 \%$ in fried vermicelli. Among MUFA, elaidic acid (trans fatty acid) was also detected in all fried vermicelli samples. The trans fatty acids in fried vermicelli was quantified in the range of $12.0-24.3 \%$, highest and lowest value was found in fried vermicelli-17 and vermicelli-2, respectively, which indicated that fried vermicelli prepared in hydrogenated oil. Nutritional values of PUFA are very important because of essential FAs. The amount of PUFA in fried vermicelli samples was observed comparatively in less quantity (0.66-5.99\%). Linoleic acid (C18:2) was the main fatty acid in PUFA group.

Table 2 shows the fatty acid ratios and nutritive values of the fried vermicelli. SFA/UFA ratio showed co-relation of two main fatty acid groups. The value of this ratio was found between $0.72-1.92$, indicated that SFA proportion present in higher amount. It has been reported that high ratio increases plasma very low density lipoprotein (VLDL) lipids and reduces the hepatic hypertriglyceridemic effect [17]. The minimum value of cis PUFA/SFA ratio set as 0.45 by British Department of Health, UK [18]. In this study, cis PUFA/SFA ratio were found lower than recommended limits in fried vermicelli samples which are not good for health in terms of CVD. Highest value of cis PUFA/SFA was found in fried vermicelli-12 (0.103) and minimum in fried vermicelli-3 (0.013). Trans $\mathrm{FA} /$ cis FA ratio is also a very important parameter which shows the degree of conversion of cis to trans configuration. In current study the ratio was found between 0.1 and 1.81 . Higher values of this ratio indicated higher proportion of hydrogenated oil. cis PUFA/SFA + TFA and cis MUFA + cis PUFA/SFA + TFA are commonly used to express nutritional value of edible oils and fats [19]. In this study the values of cis PUFA/SFA + TFA were found between 0.01 and 0.097 , whereas cis MUFA + cis PUFA/SFA + TFA ratio were found in the range of 27.7 to 37.05 in fried-vermicelli. 
Fatty acid composition of fried vermicelli

Table 1

\begin{tabular}{|c|c|c|c|c|c|c|c|c|}
\hline $\begin{array}{c}\text { Fried } \\
\text { vermicelli } \\
\text { Sample } \\
\text { No. } \\
\end{array}$ & C 12:0 & C 14:0 & C 16:0 & C18:2 & $\begin{array}{c}\text { C18: } 1 \\
\text { Cis }\end{array}$ & $\begin{array}{c}\text { C18:1 } \\
\text { trans }\end{array}$ & C18:0 & C 20:0 \\
\hline 1 & - & $\begin{array}{c}0.14 \\
\pm 0.01 \\
\end{array}$ & $\begin{array}{c}41.5 \\
\pm 0.00 \\
\end{array}$ & $\begin{array}{c}2.73 \\
\pm 0.00 \\
\end{array}$ & $\begin{array}{c}37.0 \\
\pm 0.01\end{array}$ & $\begin{array}{c}12.2 \\
\pm 0.00\end{array}$ & $\begin{array}{c}6.38 \\
\pm 0.00 \\
\end{array}$ & - \\
\hline 2 & - & $\begin{array}{c}0.54 \\
\pm 0.01\end{array}$ & $\begin{array}{c}41.7 \\
\pm 0.01\end{array}$ & $\begin{array}{c}2.14 \\
\pm 0.02\end{array}$ & $\begin{array}{c}35.1 \\
\pm 0.07\end{array}$ & $\begin{array}{c}12.0 \\
\pm 0.01\end{array}$ & $\begin{array}{c}8.49 \\
\pm 0.01\end{array}$ & - \\
\hline 3 & $\begin{array}{c}0.03 \\
\pm 0.01\end{array}$ & $\begin{array}{c}0.25 \\
\pm 0.03\end{array}$ & $\begin{array}{c}1.6 \\
\pm 0.01\end{array}$ & $\begin{array}{c}0.66 \\
\pm 0.01\end{array}$ & $\begin{array}{c}33.0 \\
\pm 0.04\end{array}$ & $\begin{array}{c}13.8 \\
\pm 0.01\end{array}$ & $\begin{array}{c}10.6 \\
\pm 0.07\end{array}$ & $\begin{array}{c}0.07 \\
\pm 0.00\end{array}$ \\
\hline 4 & - & $\begin{array}{c}0.52 \\
\pm 0.02 \\
\end{array}$ & $\begin{array}{c}37.3 \\
\pm 0.01 \\
\end{array}$ & $\begin{array}{c}3.22 \\
\pm 0.05 \\
\end{array}$ & $\begin{array}{c}30.6 \\
\pm 0.01 \\
\end{array}$ & $\begin{array}{c}19.4 \\
\pm 0.01 \\
\end{array}$ & $\begin{array}{c}8.91 \\
\pm 0.02 \\
\end{array}$ & - \\
\hline 5 & - & $\begin{array}{c}0.66 \\
\pm 0.01 \\
\end{array}$ & $\begin{array}{c}38.8 \\
\pm 0.02 \\
\end{array}$ & $\begin{array}{c}5.99 \\
\pm 0.02 \\
\end{array}$ & $\begin{array}{c}32.5 \\
\pm 0.00 \\
\end{array}$ & $\begin{array}{c}14.5 \\
\pm 0.01 \\
\end{array}$ & $\begin{array}{c}7.54 \\
\pm 0.01 \\
\end{array}$ & - \\
\hline 6 & $\begin{array}{c}0.02 \\
\pm 0.01\end{array}$ & $\begin{array}{c}0.26 \\
\pm 0.05\end{array}$ & $\begin{array}{c}34.6 \\
\pm 0.01\end{array}$ & $\begin{array}{c}1.97 \\
\pm 0.01\end{array}$ & $\begin{array}{c}32.0 \\
\pm 0.01\end{array}$ & $\begin{array}{c}23.4 \\
\pm 0.05\end{array}$ & $\begin{array}{c}7.60 \\
\pm 0.01\end{array}$ & $\begin{array}{c}0.12 \\
\pm 0.01\end{array}$ \\
\hline 7 & $\begin{array}{c}0.18 \\
\pm 0.01\end{array}$ & $\begin{array}{c}0.86 \\
\pm 0.00\end{array}$ & $\begin{array}{c}40.6 \\
\pm 0.00\end{array}$ & $\begin{array}{c}3.92 \\
\pm 0.00\end{array}$ & $\begin{array}{c}30.2 \\
\pm 0.01\end{array}$ & $\begin{array}{c}15.4 \\
\pm 0.01\end{array}$ & $\begin{array}{c}8.58 \\
\pm 0.00\end{array}$ & $\begin{array}{c}0.24 \\
\pm 0.05\end{array}$ \\
\hline 8 & $\begin{array}{c}0.16 \\
\pm 0.01\end{array}$ & $\begin{array}{c}0.89 \\
\pm 0.01\end{array}$ & $\begin{array}{c}42.1 \\
\pm 0.00\end{array}$ & $\begin{array}{c}3.90 \\
\pm 0.01\end{array}$ & $\begin{array}{c}28.8 \\
\pm 0.03\end{array}$ & $\begin{array}{c}17.2 \\
\pm 0.01\end{array}$ & $\begin{array}{c}6.73 \\
\pm 0.00\end{array}$ & $\begin{array}{c}0.27 \\
\pm 0.01\end{array}$ \\
\hline 9 & $\begin{array}{c}0.30 \\
\pm 0.01\end{array}$ & $\begin{array}{c}0.79 \\
\pm 0.01\end{array}$ & $\begin{array}{c}40.8 \\
\pm 0.06\end{array}$ & $\begin{array}{c}3.58 \\
\pm 0.04\end{array}$ & $\begin{array}{c}29.3 \\
\pm 0.00\end{array}$ & $\begin{array}{c}16.7 \\
\pm 0.06\end{array}$ & $\begin{array}{c}8.60 \\
\pm 0.02\end{array}$ & - \\
\hline 10 & $\begin{array}{c}0.55 \\
\pm 0.01\end{array}$ & - & $\begin{array}{c}47.5 \\
\pm 0.00\end{array}$ & $\begin{array}{c}1.56 \\
\pm 0.01\end{array}$ & $\begin{array}{c}27.2 \\
\pm 0.01\end{array}$ & $\begin{array}{c}18.4 \\
\pm 0.01\end{array}$ & $\begin{array}{c}4.76 \\
\pm 0.05\end{array}$ & - \\
\hline 11 & - & $\begin{array}{c}0.68 \\
\pm 0.02\end{array}$ & $\begin{array}{c}39.6 \\
\pm 0.01\end{array}$ & $\begin{array}{c}2.81 \\
\pm 0.03\end{array}$ & $\begin{array}{c}28.3 \\
\pm 0.05\end{array}$ & $\begin{array}{c}22.9 \\
\pm 0.01\end{array}$ & $\begin{array}{c}5.78 \\
\pm 0.01\end{array}$ & - \\
\hline 12 & - & $\begin{array}{c}0.40 \\
\pm 0.02\end{array}$ & $\begin{array}{c}38.5 \\
\pm 0.01\end{array}$ & $\begin{array}{c}4.74 \\
\pm 0.01\end{array}$ & $\begin{array}{c}30.0 \\
\pm 0.01\end{array}$ & $\begin{array}{c}19.3 \\
\pm 0.03\end{array}$ & $\begin{array}{c}6.94 \\
\pm 0.00\end{array}$ & $\begin{array}{c}0.15 \\
\pm 0.04\end{array}$ \\
\hline 13 & - & $\begin{array}{c}0.48 \\
\pm 0.01 \\
\end{array}$ & $\begin{array}{c}38.8 \\
\pm 0.04 \\
\end{array}$ & $\begin{array}{c}2.86 \\
\pm 0.01 \\
\end{array}$ & $\begin{array}{c}28.5 \\
\pm 0.00 \\
\end{array}$ & $\begin{array}{c}23.9 \\
\pm 0.01 \\
\end{array}$ & $\begin{array}{c}5.43 \\
\pm 0.01 \\
\end{array}$ & - \\
\hline 14 & - & $\begin{array}{c}0.15 \\
\pm 0.05\end{array}$ & $\begin{array}{c}40.4 \\
\pm 0.05\end{array}$ & $\begin{array}{c}1.40 \\
\pm 0.00\end{array}$ & $\begin{array}{c}31.6 \\
\pm 0.05\end{array}$ & $\begin{array}{c}20.5 \\
\pm 0.02\end{array}$ & $\begin{array}{c}5.88 \\
\pm 0.01\end{array}$ & - \\
\hline 15 & - & $\begin{array}{c}0.37 \\
\pm 0.01\end{array}$ & $\begin{array}{c}37.0 \\
\pm 0.00\end{array}$ & $\begin{array}{c}2.15 \\
\pm 0.01\end{array}$ & $\begin{array}{c}31.6 \\
\pm 0.01\end{array}$ & $\begin{array}{c}22.0 \\
\pm 0.00\end{array}$ & $\begin{array}{c}6.74 \\
\pm 0.05\end{array}$ & $\begin{array}{c}0.18 \\
\pm 0.05\end{array}$ \\
\hline 16 & $\begin{array}{c}0.02 \\
\pm 0.01\end{array}$ & $\begin{array}{c}0.26 \\
\pm 0.06\end{array}$ & $\begin{array}{c}37.3 \\
\pm 0.02\end{array}$ & $\begin{array}{c}1.91 \\
\pm 0.05\end{array}$ & $\begin{array}{c}33.6 \\
\pm 0.02\end{array}$ & $\begin{array}{c}20.4 \\
\pm 0.01\end{array}$ & $\begin{array}{c}6.49 \\
\pm 0.01\end{array}$ & $\begin{array}{c}0.06 \\
\pm 0.00\end{array}$ \\
\hline 17 & $\begin{array}{c}0.03 \\
\pm 0.01 \\
\end{array}$ & $\begin{array}{c}0.38 \\
\pm 0.01 \\
\end{array}$ & $\begin{array}{c}39.1 \\
\pm 0.01 \\
\end{array}$ & $\begin{array}{c}1.30 \\
\pm 0.02 \\
\end{array}$ & $\begin{array}{c}28.5 \\
\pm 0.02\end{array}$ & $\begin{array}{c}24.3 \\
\pm 0.04\end{array}$ & $\begin{array}{c}6.38 \\
\pm 0.06 \\
\end{array}$ & $\begin{array}{c}0.07 \\
\pm 0.01\end{array}$ \\
\hline 18 & - & $\begin{array}{c}0.69 \\
\pm 0.01\end{array}$ & $\begin{array}{c}41.9 \\
\pm 0.00\end{array}$ & $\begin{array}{c}2.48 \\
\pm 0.02\end{array}$ & $\begin{array}{c}29.4 \\
\pm 0.01\end{array}$ & $\begin{array}{c}16.5 \\
\pm 0.01\end{array}$ & $\begin{array}{c}9.06 \\
\pm 0.01\end{array}$ & - \\
\hline
\end{tabular}


Table 2

Fatty acid groups and fatty acids ratios of fried vermicelli (SFA, cis MUFA, trans MUFA, total MUFA, cis PUFA, SFA+TFA, MUFA+PUFA, cis MUFA+PUFA, SFA/UFA, cis PUFA/SFA, trans MUFA+ cis MUFA, cis PUFA / SFA+TFA, cis MUFA+PUFA/SFA+TFA)

\begin{tabular}{|c|c|c|c|c|c|c|c|c|c|c|c|c|c|}
\hline 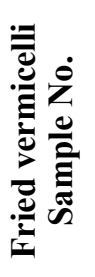 & 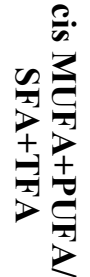 & 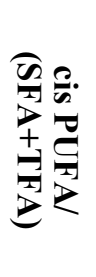 & 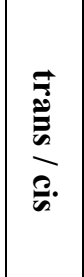 & 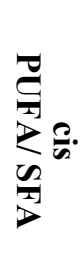 & 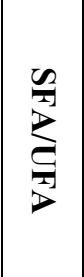 & 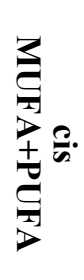 & 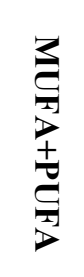 & 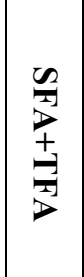 & 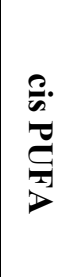 & $\begin{array}{l}\overrightarrow{0} \\
\stackrel{0}{0} \\
3 \\
2 \\
2 \\
2\end{array}$ & 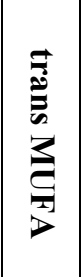 & 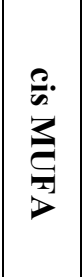 & $\begin{array}{l}\mathscr{\Omega} \\
\mathbb{D} \\
D\end{array}$ \\
\hline 1 & .05 & .045 & 0.308 & 0.057 & 0.923 & 39.74 & 51.99 & 60.26 & 2.73 & 49.30 & 12.26 & 37.01 & 48.00 \\
\hline 2 & 35.15 & 0.034 & 0.322 & 0.042 & 1.030 & 37.26 & 49.25 & 62.72 & 2.14 & 47.1 & 11.99 & 35.12 & 50.73 \\
\hline 3 & 33.04 & 0.010 & 0.410 & 0.013 & 1.105 & 33.69 & 47.50 & 66.31 & 0.66 & 46.8 & 13.81 & 3 & 52.50 \\
\hline 4 & 30.70 & 0.049 & 0.571 & 0.069 & 0.878 & 33.87 & 53.23 & 66.12 & 3.22 & 50.0 & 19.36 & 30.65 & 46.76 \\
\hline 5 & 32.59 & 0.097 & 0.377 & 0.128 & 0.887 & 38.48 & 53.00 & 61.51 & 5.99 & 47.0 & 14.52 & 32.49 & 46.99 \\
\hline 6 & 32.05 & 0.030 & 0.688 & 0.046 & 0.743 & 33.99 & 57.36 & 66.01 & 1.97 & 55.4 & 23.37 & 32.02 & 42.64 \\
\hline 7 & 30.22 & 0.059 & 0.452 & 0.078 & 1.020 & 34.08 & 49.49 & 65.92 & 3.92 & 45.6 & 15.41 & 30.16 & 50.51 \\
\hline 8 & 28.81 & 0.058 & 0.528 & 0.078 & 1.004 & 32.66 & 49.89 & 67.34 & 3.90 & 46.0 & 17.23 & 28.75 & 50.11 \\
\hline 9 & 29.36 & 0.053 & 0.507 & 0.071 & 1.018 & 32.89 & 49.55 & 67.11 & 3.58 & 46.0 & 16.67 & 29.31 & 50.45 \\
\hline 10 & 27.27 & 0.022 & 0.638 & 0.030 & 1.118 & 28.81 & 47.20 & 71.19 & 1.56 & 45.6 & 18.39 & 27.25 & 52.80 \\
\hline 11 & 28.30 & 0.041 & 0.736 & 0.061 & 0.854 & 31.08 & 53.94 & 68.92 & 2.81 & 51.1 & 22.86 & 28.26 & 46.06 \\
\hline 12 & 30.04 & 0.072 & 0.557 & 0.103 & 0.850 & 34.71 & 54.05 & 65.29 & 4.74 & 49.3 & 19.34 & 29.97 & 45.95 \\
\hline 13 & 28.52 & 0.042 & 0.764 & 0.064 & 0.809 & 31.34 & 55.28 & 68.66 & 2.86 & 52.4 & 23.94 & 28.49 & 44.72 \\
\hline 14 & 31.67 & 0.021 & 0.619 & 0.030 & 0.868 & 33.05 & 53.53 & 66.95 & 1.41 & 52.1 & 20.47 & 31.64 & 46.47 \\
\hline 15 & 31.60 & 0.032 & 0.652 & 0.048 & 0.795 & 33.72 & 55.71 & 66.28 & 2.15 & 53.6 & 21.98 & 31.57 & 44.29 \\
\hline 16 & 33.64 & 0.030 & 0.573 & 0.043 & 0.790 & 35.52 & 55.88 & 64.48 & 1.91 & 53.9 & 20.35 & 33.61 & 44.12 \\
\hline 17 & 28.50 & 0.018 & 0.814 & 0.028 & 0.850 & 29.78 & 54.04 & 70.22 & 1.30 & 52.7 & 24.26 & 28.48 & 45.95 \\
\hline 18 & 29.43 & 0.036 & 0.518 & 0.048 & 1.067 & 31.87 & 48.37 & 68.13 & 2.48 & 45.9 & 16.50 & 29.40 & 51.63 \\
\hline
\end{tabular}

\section{ATR-FTIR spectra of fried vermicelli}

The representative FTIR spectra of extracted oil of fried vermicelli are shown in Figure 1A. The assignments of the bands corresponding to the stretching vibration modes are usually easier than the assignments of the bands corresponding to the bending vibration modes due to overlap [20]. The stretching vibration band at $3004 \mathrm{~cm}^{-1}$ and two intense bands at $2920 \mathrm{~cm}^{-1}$ and $2851 \mathrm{~cm}^{-1}$ correspond to the cis double-bonds and asymmetric and symmetric stretching vibration of aliphatic $\mathrm{C}-\mathrm{H}_{2}$ functional groups, respectively.

Intense band of carbonyl functional group of triglycerides appear at $1742 \mathrm{~cm}^{-1}$, the aliphatic groups of the $\mathrm{CH}_{2}$ and $\mathrm{CH}_{3}$ shows a band near $1465 \mathrm{~cm}^{-1}$ because of the bending vibrations, whereas the rocking vibrations of $\mathrm{CH}$ band at $1418 \mathrm{~cm}^{-1}$ appeared due to the cis-olefins and $1377 \mathrm{~cm}^{-1}$ band is due to bending vibrations of $\mathrm{CH}_{2}$ groups. Stretching vibrations of $\mathrm{C}-\mathrm{O}$ ester group typically shows four bands at 1235, 1161, 1116 and 1097 $\mathrm{cm}^{-1}$ along with $\mathrm{CH}_{2}$ group associated with bending vibrations at first two frequencies i.e. 
$1235 \mathrm{~cm}^{-1}$ and $1161 \mathrm{~cm}^{-1}$. Characteristic band of isolated trans olefins at $966 \mathrm{~cm}^{-1}$ appears due to the bending vibrations of $\mathrm{C}-\mathrm{H}$ out of plane deformation, while the band at $721 \mathrm{~cm}^{-1}$ is due to the rocking vibration of out of plane cis olefins overlapping of $\mathrm{CH}_{2}$.

\section{FTIR spectral pre-treatment and data correction}

For the FTIR quantification of various fatty acid groups and their ratios, different regions of the mid IR range were used to construct suitable model using 1st and 2nd derivative spectra in order to improve regression values as compare to the pure absorbance signal. Figure 1A, 1B and 1C shows normal, 1st derivative and 2nd derivative spectra of extracted fried vermicelli oil, respectivley.

\section{PLS models}

Table 3A and Table 3B illustrates the quality parameters based on Fig 2A and Fig 2B including selected spectral region, baseline type, factors, R2 value and RMSEC of models to predict groups such as SFA, MUFA, cis-MUFA, PUFA, TFA and fatty acid ratios. The number of factors used in PLS models were auto selected by TQ software in regression analysis based on attaining minimum predicted residual error of sum of squares (PRESS) value. For the development of calibration model partial least square (PLS) regression was applied, whereas the obtained GC-MS values of SFA, MUFA, cis-MUFA, PUFA, TFA and their ratios were used as reference to establish correlation between actual GC-MS and predicted FTIR values. For each PLS model 18 samples were used as calibration points. It was found that difference between actual and predicted values of SFA, MUFA, cisMUFA, PUFA, TFA and their ratios were very small which indicates successfulness of developed PLS models.

Minimum three models were developed by selecting different spectral regions for each fatty acids groups and ratios. For SFA model best results were achieved in the regions of 2970-2820 \& 1400-1200 $\mathrm{cm}^{-1}$ with R2 and RMSEC value of 0.999 and 0.112 , respectively. Model developed for total MUFA showed higher R2 value 0.99916 in the region of 3000-3050 $\mathrm{cm}^{-1}$ using linear removed baseline with RMSEC 0.132 . On the other hand, individually cis and trans MUFA models demonstrated good results with linear removed baseline in the region of $3050-2850 \mathrm{~cm}^{-1}$ and 2nd derivative in the spectral range of $980-945 \mathrm{~cm}^{-1}$. The high R2 values were achieved in both models 0.999 and 0.991 with minimum RMSEC value 0.00984 and 0.504 , respectively. For the quantification of cis PUFA in fried vermicelli, best model was developed in the range of $2989-2869 \mathrm{~cm}^{-1}$ using linear removed baseline type with R2 and RMSEC values of 0.999 and 0.031 , respectively. The developed PLS model for fatty acid ratios including SFA+TFA (28802815 \& 980-945), MUFA+PUFA (3010-2880), cis MUFA+PUFA (3050-2650 \& 16501800), SFA/UFA (3010-2880), cis PUFA/SFA (2950-2800 \& 679-786), trans MUFA/cis MUFA (2950-2880 \& 980-945) and cis MUFA+PUFA/SFA+TFA (3050-2800 \& 15201000) showed R2 values of 0.999 except in cis PUFA/(SFA+TFA) (2950-2800 \& 980 945) model which has R2 value of 0.997 with the maximum RMSEC value of 0.092 in SFA+TFA model and minimum RMSEC was found to be $0.11 \mathrm{e}-3$ in cis PUFA/ SFA model. Table 4A and Table 4B show the results of fatty acids groups and fatty acids ratios respectively, obtained from GC-MS and FTIR with difference between them. In all the developed FTIR models, there is very little difference compared to GC-MS results. 

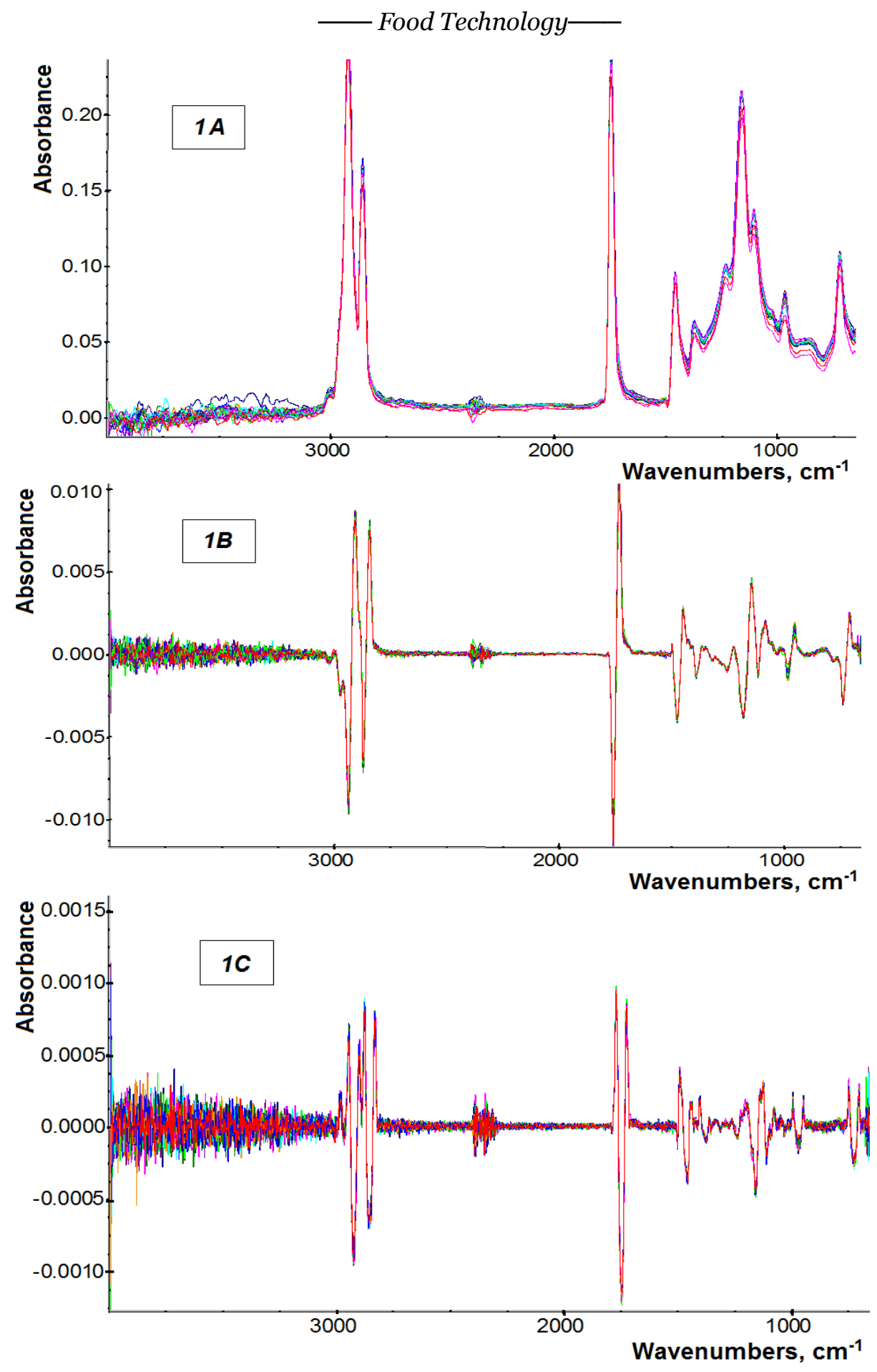

Figure 1.

A - Normal FTIR spectra of fried vermicelli; B - $1^{\text {st }}$ derivative spectra; $\mathrm{C}-2^{\text {nd }}$ derivative spectra of all the samples 
Table 3A

FTIR PLS correlation model of fatty acid groups

\begin{tabular}{|c|c|c|c|c|c|}
\hline \multirow{2}{*}{ Fatty acid } & Spectral region $\mathbf{~ c m}^{-\mathbf{1}}$ & Baseline type & Factors & $\mathbf{R}^{\mathbf{2}}$ & RMSEC \\
& & & & & \\
\hline \multirow{3}{*}{ SFA } & $3050-650$ & None & 2 & 0.818 & 1.74 \\
& $2950-2820 \& 1125-1080$ & None & 4 & 0.956 & 0.888 \\
& $\mathbf{2 9 7 0 - 2 8 2 0 \& 1 4 0 0 - 1 2 0 0}$ & None & $\mathbf{7}$ & $\mathbf{0 . 9 9 9}$ & $\mathbf{0 . 1 1 2}$ \\
\hline \multirow{3}{*}{ cis MUFA } & $3050-2950$ & None & 8 & 0.999 & 0.0357 \\
& $3020-650$ & None & 3 & 0.885 & 1.15 \\
& $\mathbf{3 0 5 0 - 2 8 5 0}$ & Linear & $\mathbf{1 0}$ & $\mathbf{0 . 9 9 9}$ & $\mathbf{0 . 0 0 9}$ \\
& & removed & & & \\
\hline \multirow{4}{*}{ trans MUFA } & $980-945$ & None & 4 & 0.984 & 0.674 \\
& $980-945$ & $1^{\text {st }}$ Derivative & 4 & 0.988 & 0.605 \\
& $\mathbf{9 8 0 - 9 4 5}$ & Derivative & $\mathbf{5}$ & $\mathbf{0 . 9 9 1}$ & $\mathbf{0 . 5 0 4}$ \\
\hline \multirow{3}{*}{ Total } & $3050-650$ & None & 3 & 0.958 & 1.04 \\
MUFA & $4000-650$ & Linear & 2 & 0.959 & 1.03 \\
& $\mathbf{3 0 5 0 - 3 0 0 0}$ & Removed & & $\mathbf{0 . 9 9 9}$ & $\mathbf{0 . 1 3 2}$ \\
& & None & 5 & 0.984 & 0.229 \\
cis PUFA & $2989-2869$ & None & 1 & 0.759 & 0.839 \\
& $4000-650$ & Linear & $\mathbf{8}$ & $\mathbf{0 . 9 9 9}$ & $\mathbf{0 . 0 3 1}$ \\
& $\mathbf{2 9 8 9 - 2 8 6 9}$ & Removed & & & \\
\hline
\end{tabular}


Table 3B

FTIR PLS correlation model of fatty acid ratios

\begin{tabular}{|c|c|c|c|c|c|}
\hline Fatty acid ratios & $\begin{array}{l}\text { Spectral region } \\
\mathrm{cm}^{-1}\end{array}$ & $\begin{array}{c}\text { Baseline } \\
\text { type }\end{array}$ & Factors & $\mathbf{R}^{2}$ & RMSEC \\
\hline $\mathrm{SFA}+\mathrm{TFA}$ & $\begin{array}{c}\mathbf{2 8 8 0}-\mathbf{2 8 1 5} \& \\
\mathbf{9 8 0}-\mathbf{9 4 5} \\
3010-750 \& \\
990-945 \\
3050-2950\end{array}$ & $\begin{array}{l}\text { None } \\
\text { None } \\
\text { None }\end{array}$ & $\begin{array}{l}9 \\
2 \\
7\end{array}$ & $\begin{array}{l}\mathbf{0 . 9 9 9} \\
0.808 \\
0.999\end{array}$ & $\begin{array}{l}\mathbf{0 . 0 9 2} \\
1.570 \\
0.101\end{array}$ \\
\hline MUFA+PUFA & $\begin{array}{l}\mathbf{3 0 1 0}-\mathbf{2 8 8 0} \\
3015-2835 \\
3040-2830\end{array}$ & $\begin{array}{l}\text { None } \\
\text { None } \\
\text { None }\end{array}$ & $\begin{array}{l}7 \\
6 \\
4\end{array}$ & $\begin{array}{l}\mathbf{0 . 9 9 9} \\
0.999 \\
0.992\end{array}$ & $\begin{array}{l}\mathbf{0 . 0 9 1} \\
0.122 \\
0.383\end{array}$ \\
\hline Cis MUFA+PUFA & $\begin{array}{c}3050-2700 \& \\
1650-1800 \\
3050-2870 \& \\
1650-1800 \\
\mathbf{3 0 5 0}-\mathbf{2 6 5 0} \& \\
\mathbf{1 6 5 0}-\mathbf{1 8 0 0}\end{array}$ & $\begin{array}{c}\text { None } \\
\text { None } \\
\mathbf{1}^{\text {st }} \\
\text { Derivative }\end{array}$ & $\begin{array}{l}3 \\
3 \\
4\end{array}$ & $\begin{array}{l}0.984 \\
0.839 \\
\mathbf{0 . 9 9 9}\end{array}$ & $\begin{array}{l}0.480 \\
1.480 \\
\mathbf{0 . 0 6 0}\end{array}$ \\
\hline SFA/UFA & $\begin{array}{l}3030-2880 \\
3040-2880 \\
\mathbf{3 0 1 0}-\mathbf{2 8 8 0}\end{array}$ & $\begin{array}{l}\text { None } \\
\text { None } \\
\text { None }\end{array}$ & $\begin{array}{l}6 \\
6 \\
7 \\
\end{array}$ & $\begin{array}{l}0.999 \\
0.998 \\
\mathbf{0 . 9 9 9} \\
\end{array}$ & $\begin{array}{l}0.006 \\
0.006 \\
\mathbf{0 . 0 0 3}\end{array}$ \\
\hline cis PUFA/ SFA & $\begin{array}{c}\mathbf{2 9 5 0}-\mathbf{2 8 0 0} \& \\
\mathbf{6 7 9}-\mathbf{7 8 6} \\
2950-2800 \\
3000-2800 \\
\end{array}$ & $\begin{array}{l}\text { None } \\
\text { None } \\
\text { None }\end{array}$ & $\begin{array}{l}8 \\
5 \\
3\end{array}$ & $\begin{array}{l}\mathbf{0 . 9 9 9} \\
0.992 \\
0.929\end{array}$ & $\begin{array}{c}\mathbf{0 . 1 1}^{-3} \\
0.003 \\
0.009\end{array}$ \\
\hline trans MUFA/ cis MUFA & $\begin{array}{c}2950-2830 \& \\
980-945 \\
3050-2950 \& \\
980-945 \\
\mathbf{2 9 5 0 - 2 8 8 0} \& \\
\text { 980-945 }\end{array}$ & $\begin{array}{c}\text { None } \\
\text { None } \\
\text { Linear } \\
\text { removed }\end{array}$ & $\begin{array}{l}5 \\
3 \\
7\end{array}$ & $\begin{array}{l}0.994 \\
0.975 \\
\mathbf{0 . 9 9 9}\end{array}$ & $\begin{array}{l}0.015 \\
0.031 \\
\mathbf{0 . 0 0 6}\end{array}$ \\
\hline cis PUFA/(SFA+TFA) & $\begin{array}{c}3050-2800 \& \\
980-945 \\
2950-2800 \\
\mathbf{2 9 5 0 - 2 8 0 0} \& \\
\mathbf{9 8 0 - 9 4 5}\end{array}$ & $\begin{array}{c}\text { None } \\
\text { None } \\
\text { Linear } \\
\text { removed }\end{array}$ & $\begin{array}{l}4 \\
5 \\
6\end{array}$ & $\begin{array}{l}0.980 \\
0.992 \\
\mathbf{0 . 9 9 7}\end{array}$ & $\begin{array}{l}0.003 \\
0.002 \\
\mathbf{0 . 0 0 1}\end{array}$ \\
\hline $\begin{array}{c}\text { Cis MUFA+PUFA/ } \\
\text { SFA+TFA }\end{array}$ & $\begin{array}{c}3050-2800 \& \\
1000-900 \\
\mathbf{3 0 5 0}-\mathbf{2 8 0 0} \& \\
\mathbf{1 5 2 0}-\mathbf{1 0 0 0} \\
3050-2800 \& \\
1830-920\end{array}$ & $\begin{array}{l}\text { None } \\
\text { None } \\
\text { None }\end{array}$ & $\begin{array}{l}7 \\
8 \\
4\end{array}$ & $\begin{array}{l}0.998 \\
\mathbf{0 . 9 9 9} \\
0.961\end{array}$ & $\begin{array}{l}0.053 \\
\mathbf{0 . 0 0 6} \\
0.681\end{array}$ \\
\hline
\end{tabular}



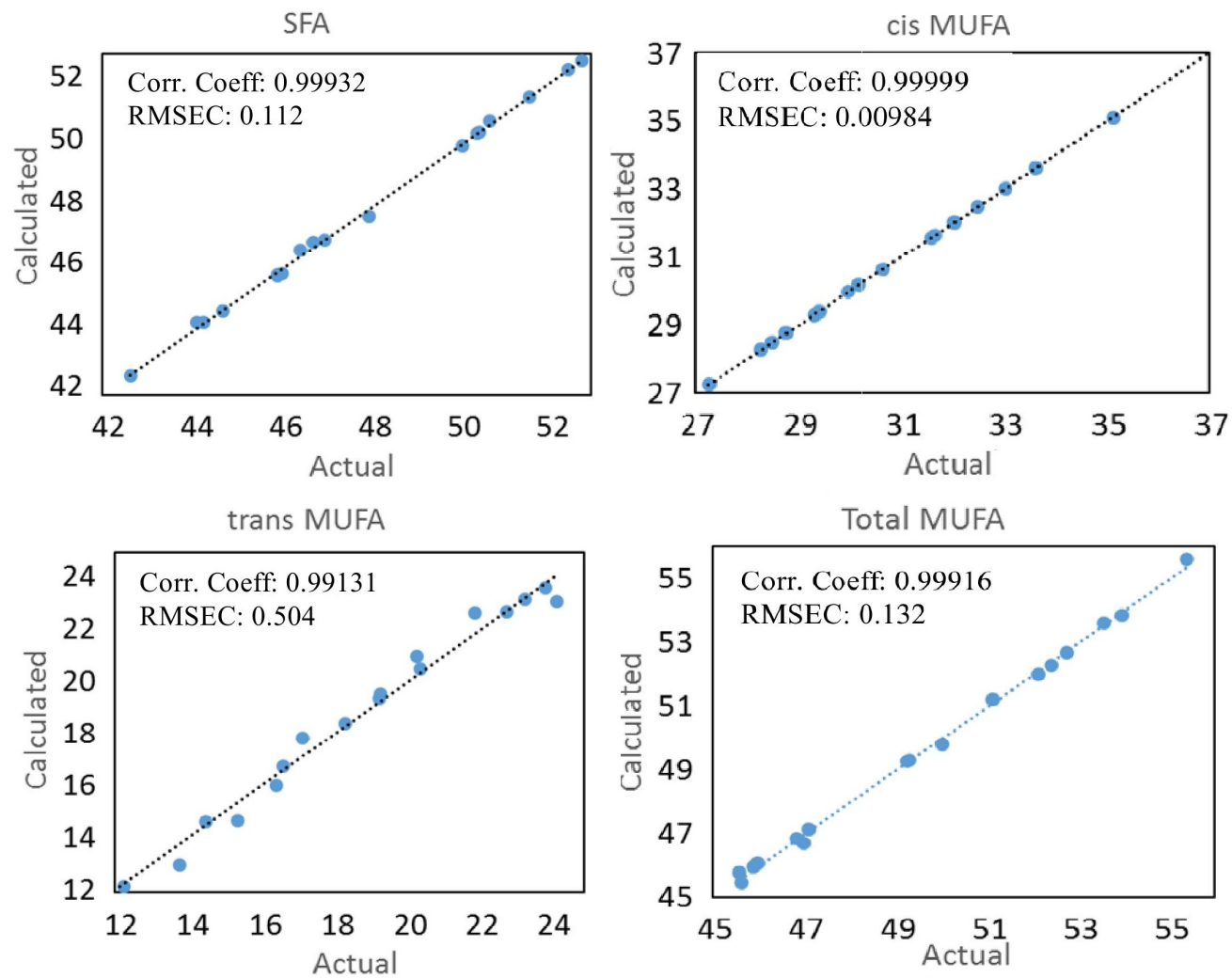

cis PUFA

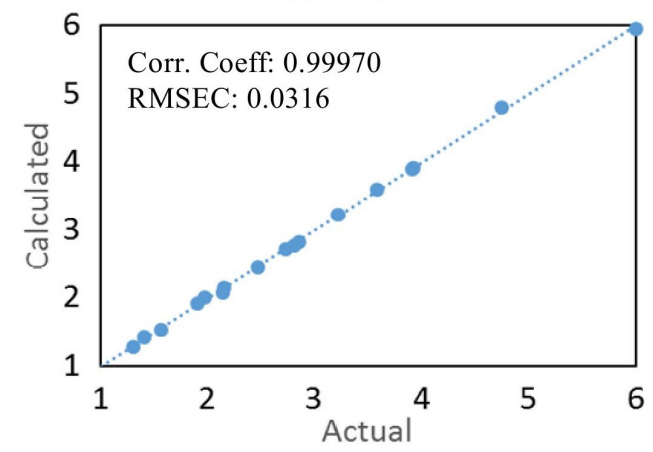

Figure 2A. PLS models for SFA, cis-MUFA, trans-MUFA, total-MUFA and total PUFA. 

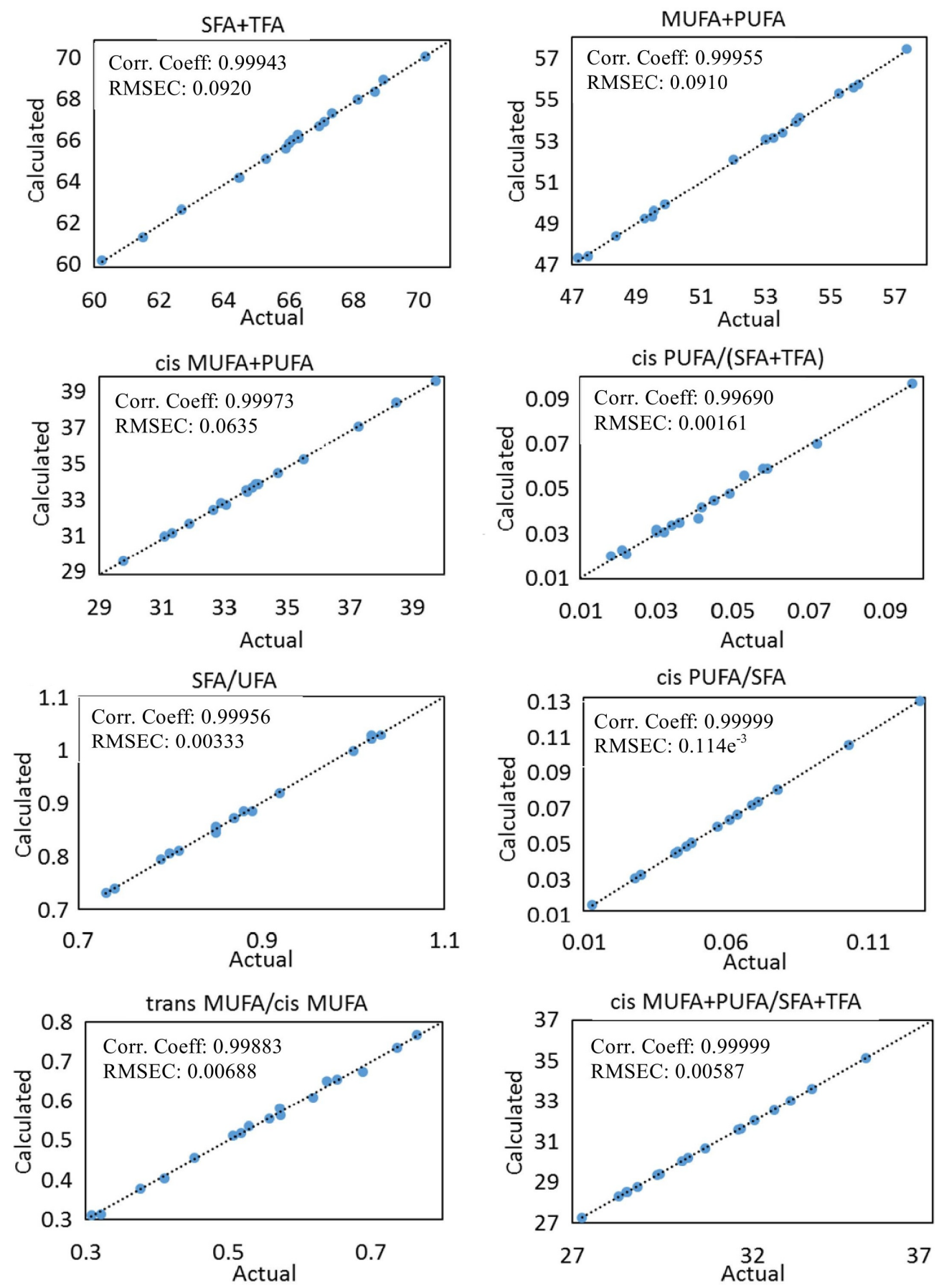

Figure 2B. PLS models for SFA+TFA, MUFA+PUFA, cis-MUFA+PUFA, SFA/TFA, cis PUFA/SFA, trans MUFA/ cis MUFA, cis PUFA/SFA+TFA and Cis MUFA+PUFA/ SFA+TFA. 
Comparative results of FTIR and GC methods for fatty acid groups

\begin{tabular}{|c|c|c|c|c|c|c|c|c|c|}
\hline $\begin{array}{c}\text { Fried } \\
\text { vermicelli } \\
\begin{array}{c}\text { Sample } \\
\text { No }\end{array}\end{array}$ & \multicolumn{3}{|c|}{ SFA } & \multicolumn{3}{c|}{ cis MUFA } & \multicolumn{3}{c|}{ trans MUFA } \\
\hline & GC & FTIR & Diff & GC & FTIR & Diff & GC & FTIR & Diff \\
\hline 1 & 48.003 & 47.769 & -0.234 & 37.010 & 37.017 & 0.007 & 12.255 & 12.235 & -0.020 \\
\hline 2 & 50.730 & 50.860 & 0.130 & 35.120 & 35.116 & -0.004 & 11.990 & 12.596 & 0.606 \\
\hline 3 & 52.498 & 52.504 & 0.006 & 33.030 & 33.024 & -0.006 & 13.808 & 13.075 & -0.733 \\
\hline 4 & 46.760 & 46.921 & 0.161 & 30.650 & 30.634 & -0.016 & 19.360 & 19.597 & 0.237 \\
\hline 5 & 46.999 & 47.006 & 0.006 & 32.492 & 32.493 & 0.001 & 14.516 & 14.696 & 0.180 \\
\hline 6 & 42.641 & 42.606 & -0.036 & 32.016 & 32.022 & 0.006 & 23.369 & 23.222 & -0.147 \\
\hline 7 & 50.508 & 50.492 & -0.016 & 30.165 & 30.156 & -0.008 & 15.412 & 14.737 & -0.675 \\
\hline 8 & 50.113 & 50.051 & -0.062 & 28.755 & 28.758 & 0.003 & 17.228 & 17.927 & 0.699 \\
\hline 9 & 50.447 & 50.450 & 0.002 & 29.310 & 29.303 & -0.007 & 16.666 & 16.838 & 0.172 \\
\hline 10 & 52.798 & 52.803 & -0.017 & 27.248 & 27.264 & 0.016 & 18.392 & 18.465 & 0.073 \\
\hline 11 & 46.062 & 45.915 & -0.147 & 28.265 & 28.255 & -0.009 & 22.859 & 22.747 & -0.112 \\
\hline 12 & 45.950 & 45.845 & -0.105 & 29.970 & 29.963 & -0.007 & 19.340 & 19.428 & 0.088 \\
\hline 13 & 44.721 & 44.683 & -0.037 & 28.487 & 28.495 & 0.009 & 23.935 & 23.659 & -0.276 \\
\hline 14 & 44.293 & 44.327 & 0.034 & 31.573 & 31.579 & 0.006 & 21.984 & 22.679 & 0.695 \\
\hline 15 & 44.124 & 44.319 & 0.195 & 33.614 & 33.606 & -0.008 & 20.354 & 21.051 & 0.698 \\
\hline 16 & 45.955 & 45.867 & -0.088 & 28.480 & 28.467 & -0.013 & 24.262 & 23.108 & -1.154 \\
\hline 17 & 46.473 & 46.675 & 0.202 & 31.646 & 31.663 & 0.018 & 20.474 & 20.562 & 0.089 \\
\hline 18 & 51.627 & 51.611 & 0.005 & 29.398 & 29.409 & 0.012 & 16.499 & 16.081 & -0.418 \\
\hline & & & & & & & & & \\
\hline
\end{tabular}

\begin{tabular}{|c|c|c|c|c|c|c|}
\hline $\begin{array}{c}\text { Fried } \\
\text { vermicelli } \\
\text { Sample No }\end{array}$ & \multicolumn{3}{|c|}{ Total MUFA } & \multicolumn{3}{c|}{ cis PUFA } \\
\hline & GC & FTIR & Diff & GC & FTIR & Diff \\
\hline 1 & 49.265 & 49.281 & 0.016 & 2.730 & 2.713 & -0.017 \\
\hline 2 & 47.110 & 47.131 & 0.021 & 2.140 & 2.077 & -0.063 \\
\hline 3 & 46.838 & 46.867 & 0.029 & 0.663 & 0.698 & 0.035 \\
\hline 4 & 50.010 & 49.810 & -0.200 & 3.220 & 3.236 & 0.016 \\
\hline 5 & 47.007 & 46.735 & -0.272 & 5.993 & 5.962 & -0.031 \\
\hline 6 & 55.386 & 55.595 & 0.209 & 1.973 & 2.011 & 0.038 \\
\hline 7 & 45.576 & 45.791 & 0.215 & 3.916 & 3.920 & 0.004 \\
\hline 8 & 45.983 & 46.093 & 0.110 & 3.905 & 3.910 & 0.005 \\
\hline 9 & 45.976 & 46.091 & 0.115 & 3.576 & 3.598 & 0.023 \\
\hline 10 & 45.640 & 45.476 & -0.164 & 1.561 & 1.539 & -0.022 \\
\hline 11 & 51.124 & 51.237 & 0.113 & 2.814 & 2.767 & -0.047 \\
\hline 12 & 49.310 & 49.317 & 0.007 & 4.740 & 4.803 & 0.063 \\
\hline 13 & 52.422 & 52.288 & -0.134 & 2.858 & 2.823 & -0.034 \\
\hline 14 & 53.557 & 53.612 & 0.055 & 2.149 & 2.162 & 0.013 \\
\hline 15 & 53.968 & 53.884 & -0.084 & 1.908 & 1.927 & 0.019 \\
\hline 16 & 52.742 & 52.706 & -0.035 & 1.304 & 1.294 & -0.010 \\
\hline 17 & 52.119 & 52.037 & -0.082 & 1.407 & 1.431 & 0.024 \\
\hline 18 & 45.897 & 45.978 & 0.081 & 2.476 & 2.459 & -0.016 \\
\hline
\end{tabular}


Table 4B

Comparative results of FTIR and GC methods for fatty acid ratios

\begin{tabular}{|c|c|c|c|c|c|c|c|c|c|c|c|c|}
\hline \multirow{2}{*}{ 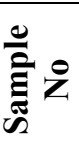 } & \multicolumn{3}{|c|}{ SFA+TFA } & \multicolumn{3}{|c|}{ MUFA+PUFA } & \multicolumn{3}{|c|}{$\begin{array}{l}\text { Cis UFA+ } \\
\text { PUFA }\end{array}$} & \multicolumn{3}{|c|}{$\begin{array}{c}\text { cis PUFA/ } \\
\text { (SFA+TFA) }\end{array}$} \\
\hline & GC & FTIR & Diff & GC & FTIR & Diff & GC & FTIR & Diff & GC & FTIR & Diff \\
\hline 1 & 60.258 & 345 & 0.087 & 51.995 & 52.093 & 0.098 & 39.740 & 39.752 & 0.012 & 0.045 & .045 & -0.000 \\
\hline 2 & 62.720 & 62.770 & 0.050 & 49.250 & 49.225 & -0.025 & 37.260 & 37.219 & -0.041 & 0.034 & 0.034 & 0.000 \\
\hline 3 & 66.306 & 66.257 & -0.049 & 47.501 & 47.419 & -0.082 & 33.693 & 33.733 & 0.040 & 0.010 & 0.009 & -0.001 \\
\hline 4 & 66.120 & 66.173 & 0.053 & 53.230 & 53.171 & -0.059 & 33.870 & 33.864 & -0.006 & 0.049 & 0.048 & -0.001 \\
\hline 5 & 61.515 & 61.469 & -0.046 & 53.000 & \begin{tabular}{|l|}
53.071 \\
\end{tabular} & 0.071 & 38.485 & 38.549 & 0.064 & 0.097 & 0.097 & 0.000 \\
\hline 6 & 66.011 & 66.035 & 0.024 & 57.358 & 57.466 & 0.108 & 33.989 & 34.060 & 0.071 & 0.030 & 0.031 & 0.001 \\
\hline 7 & 65.920 & 65.759 & -0.161 & 49.492 & 49.334 & -0.158 & 34.080 & 34.069 & -0.011 & 0.059 & 0.059 & 0.000 \\
\hline 8 & 67.341 & & 0.123 & 49.888 & 49.936 & 0.048 & 32.659 & 32.632 & -0.027 & 0.058 & .059 & 0.001 \\
\hline 9 & 67.114 & 67.062 & -0.051 & 49.552 & 49.583 & 0.031 & 32.886 & 33.034 & 0.148 & 0.053 & 0.056 & 0.003 \\
\hline 10 & 71.190 & 71.178 & -0.012 & 47.201 & 47.333 & 0.132 & 28.809 & 28.797 & -0.012 & 0.022 & 0.021 & -0.001 \\
\hline 11 & 68.921 & 69.090 & 0.169 & 53.938 & \begin{tabular}{|l|}
53.941 \\
\end{tabular} & 0.003 & 31.079 & 31.162 & 0.083 & 0.041 & 0.037 & -0.004 \\
\hline 12 & 65.290 & 65.258 & -0.032 & 54.050 & \begin{tabular}{|l|l|}
54.153 \\
\end{tabular} & 0.103 & 34.710 & 34.666 & -0.044 & 0.072 & 0.070 & -0.002 \\
\hline 13 & 68.656 & 68.530 & -0.125 & 55.279 & 55.301 & 0.022 & 31.344 & 31.357 & 0.013 & 0.042 & 0.042 & -0.000 \\
\hline 14 & 66.277 & 66.446 & 0.169 & 55.707 & 55.578 & -0.128 & 33.723 & 33.647 & -0.076 & 0.032 & 0.031 & -0.001 \\
\hline 15 & 64.478 & 64.372 & -0.106 & 55.876 & 55.735 & -0.141 & 35.522 & 35.443 & -0.079 & 0.030 & 0.032 & 0.002 \\
\hline 16 & 70.216 & 70.202 & -0.014 & 54.045 & 54.129 & 0.083 & 29.784 & 29.758 & -0.026 & 0.018 & 0.020 & 0.002 \\
\hline 17 & 66.947 & 66.874 & -0.073 & 53.527 & 53.417 & -0.110 & 33.053 & 32.930 & -0.123 & 0.021 & 0.023 & 0.002 \\
\hline 18 & 68.127 & 68.122 & -0.005 & 48.373 & 48.378 & 0.006 & 31.873 & 31.889 & 0.015 & 0.036 & 0.035 & -0.001 \\
\hline
\end{tabular}

\begin{tabular}{|c|c|c|c|c|c|c|c|c|c|c|c|c|}
\hline \multirow{2}{*}{ 离 } & \multicolumn{3}{|c|}{ SFA/UFA } & \multicolumn{3}{|c|}{$\begin{array}{l}\text { Cis PUFA / } \\
\text { SFA }\end{array}$} & \multicolumn{3}{|c|}{$\begin{array}{l}\text { Trans MUFA/ } \\
\text { cis MUFA }\end{array}$} & \multicolumn{3}{|c|}{$\begin{array}{c}\text { Cis MUFA+PUFA/ } \\
\text { SFA+TFA }\end{array}$} \\
\hline & GC & $\begin{array}{c}\text { FTI } \\
\text { R }\end{array}$ & Diff & GC & FTIR & Diff & GC & $\begin{array}{l}\text { FTI } \\
\text { R }\end{array}$ & Diff & GC & FTIR & Diff \\
\hline 1 & 0.920 & 0.917 & -0.003 & 0.057 & 0.057 & -0.000 & 0.308 & 0.311 & 0.003 & 37.050 & 37.057 & 0.007 \\
\hline 2 & 1.030 & 1.027 & -0.003 & 0.042 & 0.042 & 0.000 & 0.322 & 0.313 & -0.009 & 35.150 & 35.151 & 0.001 \\
\hline 3 & 1.100 & 1.104 & 0.004 & 0.013 & 0.013 & -0.000 & 0.410 & 0.405 & -0.005 & 33.040 & 33.039 & -0.001 \\
\hline 4 & 0.880 & 0.883 & 0.003 & 0.069 & 0.069 & 0.000 & 0.572 & 0.581 & 0.009 & 30.700 & 30.696 & -0.004 \\
\hline 5 & 0.890 & 0.883 & -0.007 & 0.128 & 0.128 & -0.000 & 0.377 & 0.379 & 0.002 & 32.590 & 32.589 & -0.001 \\
\hline 6 & 0.740 & 0.738 & -0.002 & 0.046 & 0.046 & -0.000 & 0.688 & 0.675 & -0.013 & 32.050 & 32.049 & -0.001 \\
\hline 7 & 1.020 & 1.027 & 0.007 & 0.078 & 0.078 & 0.000 & 0.452 & 0.456 & 0.004 & 30.220 & 30.215 & -0.005 \\
\hline 8 & 1.000 & 0.996 & -0.004 & 0.078 & 0.078 & -0.000 & 0.528 & 0.536 & 0.008 & 28.810 & 28.800 & -0.010 \\
\hline 9 & 1.020 & 1.019 & -0.001 & 0.071 & 0.071 & 0.000 & 0.507 & 0.513 & 0.006 & 29.360 & 29.360 & -0.000 \\
\hline 10 & 1.120 & 1.119 & -0.001 & 0.030 & 0.030 & -0.000 & 0.638 & 0.651 & 0.013 & 27.270 & 27.270 & 0.000 \\
\hline 11 & 0.850 & 0.850 & -0.000 & 0.061 & 0.061 & -0.000 & 0.736 & 0.734 & -0.002 & 28.300 & 28.316 & 0.016 \\
\hline 12 & 0.850 & 0.855 & 0.005 & 0.103 & 0.103 & -0.000 & 0.557 & 0.556 & -0.001 & 30.040 & 30.044 & 0.004 \\
\hline 13 & 0.810 & 0.810 & 0.000 & 0.064 & 0.064 & 0.000 & 0.764 & 0.768 & 0.004 & 28.520 & 28.516 & -0.004 \\
\hline 14 & 0.730 & 0.730 & 0.000 & 0.048 & 0.048 & -0.000 & 0.652 & 0.656 & 0.004 & 31.600 & 31.596 & -0.004 \\
\hline 15 & 0.800 & 0.805 & 0.005 & 0.043 & 0.043 & -0.000 & 0.573 & 0.564 & -0.009 & 33.640 & 33.640 & -0.000 \\
\hline 16 & 0.790 & 0.793 & 0.003 & 0.028 & 0.028 & 0.000 & 0.814 & 0.809 & -0.005 & 28.500 & 28.509 & 0.009 \\
\hline 17 & 0.850 & 0.843 & -0.007 & 0.030 & 0.030 & 0.000 & 0.619 & 0.610 & -0.009 & 31.670 & 31.663 & -0.007 \\
\hline 18 & 0.870 & 0.871 & 0.001 & 0.048 & 0.048 & -0.000 & 0.518 & 0.519 & 0.001 & 29.430 & 29.431 & 0.001 \\
\hline
\end{tabular}




\title{
Conclusion
}

In the present study, fried vermicelli was completely analyzed for their fatty acid profile including various groups and ratios by GC-MS. Based on results, it was concluded that fried vermicelli contain high percentage of oil ranging from 19.77 and $32.99 \%$. Fatty acid composition revealed that SFA and MUFA present in higher amount as compare to PUFA in fried vermicelli. All fried vermicelli samples showed higher trans fatty acids which indicated that samples were prepared in hydrogenated oil. Furthermore, fried vermicelli also showed less nutritional values in comparison to recommended values of trans fat and cis PUFA/SFA ratio set as $0.5 \%$ and 0.45 , respectively. FTIR spectroscopy successfully applied to quantify fatty acid groups and ratios in fried vermicelli using chemometric. The developed PLS models showed higher correlations $(>0.99)$ with less calibration error. The selected wavelength regions can be used to quantify the fatty acid group and their ratios using FTIR with least analysis time without using organic solvents.

\author{
Acknowledgements \\ The National Centre of Excellence in Analytical Chemistry, \\ University of Sindh, Jamshoro, Pakistan is gratefully \\ acknowledged for providing the financial support and \\ laboratory facilities.
}

\section{References}

1. Burlingame B., Nishida C., Uauy R., Weisell R. (2009), Fats and fatty acids in human nutrition: introduction, Annals of Nutrition and Metabolism, 55(1-3), pp. 5-7.

2. Simopoulos, A. P. (2002), The importance of the ratio of omega-6/omega-3 essential fatty acids, Biomedicine \& pharmacotherapy, 56(8), pp. 365-379.

3. Mozaffarian D., Micha R., Wallace S. (2010), Effects on coronary heart disease of increasing polyunsaturated fat in place of saturated fat: a systematic review and metaanalysis of randomized controlled trials, PLoS Medicine, 7(3), e1000252, DOI: 10.1371/journal.pmed.1000252

4. Gillingham L G., Harris-Janz S., Jones P J. (2011), Dietary monounsaturated fatty acids are protective against metabolic syndrome and cardiovascular disease risk factors, Lipids, 46(3), pp. 209-228.

5. Poudyal H., Panchal S K., Diwan V., Brown L. (2011), Omega-3 fatty acids and metabolic syndrome: Effects and emerging mechanisms of action, Progress in Lipid Research, 50(4), pp. 372-387.

6. Erkkila A., de Mello V D., Risérus U., Laaksonen D E. (2008), Dietary fatty acids and cardiovascular disease: an epidemiological approach, Progress in Lipid Research, 47(3), pp. 172-187.

7. Oh K., Hu FB., Manson J E., Stampfer M J., Willett W C. (2005), Dietary fat intake and risk of coronary heart disease in women: 20 years of follow-up of the nurses' health study, American Journal of Epidemiology, 161(7), pp. 672-679.

8. Czernichow S., Thomas D., Buckert E. (2010), n-6 Fatty acids and cardiovascular health: A review of the evidence for dietary intake recommendations, British Journal of Nutrition, 104(06), pp. 788-796. 
9. Shahidi F., Wanasundara U N. (2002), Methods for measuring oxidative rancidity in fats and oils, Food lipids: Chemistry, Nutrition and Biotechnology, pp. 387-403.

10. Eckel R H., Borra S., Lichtenstein A H., Yin-Piazza S Y. (2007), Understanding the complexity of trans fatty acid reduction in the American Diet American Heart Association trans Fat conference 2006: report of the trans fat conference planning group, Circulation, 115(16), pp. 2231-2246.

11. Food and Drug Administration F.D.A. (2003), Food labeling: trans fatty acids in nutrition labeling, nutrient content claims, and health claims. Final rule, Federal Register, 68(133), pp. 41433.

12. Uauy R., Aro A., Clarke R., L'abbé M R., Mozaffarian D., Skeaff C M., Stender S., Tavella M. (2009), WHO Scientific Update on trans fatty acids: summary and conclusions, European Journal of Clinical Nutrition, 63, pp. S68-S75.

13. Food and Drug Administration F.D.A. (2015), The FDA takes step to remove artificial trans fats in processed foods, Available at:

http://www.fda.gov/NewsEvents/Newsroom/PressAnnouncements/ucm451237.html accessed 12.02.2016.

14. AOAC (1997), Official methods of analysis. 16th ed, Gaithersburg, Maryland.

15. IUPAC (1979), Standard methods for the analysis of oils, fats and derivatives (6th ed.), Pergamon Press, Oxford, pp. 96-98.

16. Kandhro A., Sherazi S T H., Mahesar S A., Bhanger M I., Talpur M Y., Arain S. (2008), Monitoring of fat content, free fatty acid and fatty acid profile including trans fat in Pakistani biscuits, Journal of the American Oil Chemists' Society, 85(11), pp. 1057-1061.

17. Chang N W., Ten Wu C., Chen F N., Huang P C. (2004), High polyunsaturated and monounsaturated fatty acid to saturated fatty acid ratio increases plasma very low density lipoprotein lipids and reduces the hepatic hypertriglyceridemic effect of dietary cholesterol in rats, Nutrition research, 24(1), pp. 73-83.

18. HMSO, UK Department of Health. (1994), Nutritional aspects of cardiovascular disease. London, Report on Health and Social Subjects, 46, pp. 37-46

19. Alonso L., Fraga M J., Juarez M. (2000), Determination of trans fatty acids and fatty acid profile in margarines marketed in Spain, American Oil Chemists' Society, 77(2), pp. 131-136.

20. Guillen M D., Cabo N. (1997), Infrared spectroscopy in the study of edible oils and fats, Journal of the Science of Food and Agriculture, 75(1), pp. 1-11. 